\title{
Interaction of display mode and character size in vibrotactile letter recognition
}

\author{
JACK M. LOOMIS \\ University of California, Santa Barbara, California 93106
}

\begin{abstract}
Research with the Tactile Vision Substitution System, a device that converts optical patterns into vibrotactile patterns for display against the back, has shown that a simple pattern, such as a letter, is more easily recognized when presented sequentially than when presented in entirety all at once. In recent work with the Optacon, another such device using the surface of the finger for display, Craig (Note 2) has found quite the opposite result over an extensive range of pattern durations. The present study shows that the superiority of sequential presentation can be obtained with the Optacon if letters considerably smaller than usual are displayed. Sequential presentation is advantageous when the pattern information essential for recognition exceeds the spatial bandwidth of the sensory surface, whether it be the finger, back, or other skin surface. In the normal usage of the Optacon, however, it appears that the spatial bandwidth of the finger suffices for the recognition of large roman letters.
\end{abstract}

Research with the Tactile Vision Substitution System (TVSS), an electronic device that converts high-contrast optical patterns (e.g., letters) into vibrotactile patterns for display against the back, has shown that a simple pattern is more easily recognized when presented sequentially than when presented in entirety all at once. Beauchamp, Matheson, and Scadden (1971) found that drawing simple patterns out with a single point of light yielded better recognition performance than panning the same patterns with the camera. ApkarianStielau and Loomis (1975) and Loomis (1974) demonstrated that letters scanned horizontally by a moving vertical slit were more easily recognized than letters simply flashed on the tactile display. More recently, Saida, Shimizu, and Wake (Note 1) have shown a very sizable superiority of sequential presentation over static presentation, using a tactile display against the belly. Loomis (1974) interpreted the superiority of sequential presentation as a consequence of the limited spatial resolution of the cutaneous sense, reasoning that stimuli that fail to be resolved spatially when presented simultaneously often can be accurately perceived when presented sequentially. Extending his argument to include the finger, Loomis argued that contrary results obtained with the Optacon, a device like the TVSS used for reading by the blind, were understandable for reasons relating to the very rapid rates at which the letters were sequentially presented.

In a recent study with the Optacon, Craig (Note 2) has shown that slit-scan and other modes of sequential presentation lead not to better, but to poorer recognition performance over an extensive range of pattern durations. This result suggests that the limited-resolution

This work was supported by Grant 1 R01 NS15129 from NINCDS. The author thanks Pauline McNulty for her assistance. hypothesis does not apply to the recognition of letters presented to the finger with the Optacon, at least not in its normal usage. However, one would still expect that if letters are reduced to a size such that spatial resolution does limit their recognition, the earlier result of superior performance with sequential presentation would obtain with the Optacon as well. The present experiment tested this expectation by comparing slit-scan and static presentation modes using smaller than usual letters; for purposes of comparison, normal-sized letters were studied as well.

\section{METHOD}

\section{Apparatus}

The apparatus used here was similar in concept to that employed by Loomis (1974), the major difference being that the Optacon (Model R1D) was used rather than the TVSS. Relatively large photographic negatives of letters, mounted vertically, were transilluminated by diffuse light and imaged by an auxiliary lens (focal length $=29 \mathrm{~mm}$ ) onto the object plane of the fixed-focus Optacon camera. The letters either were flashed for $1.5 \mathrm{sec}$ using a shutter (static mode of presentation) or were scanned by a vertical slit that was positioned just in front of the letter and moved from left to right in $1.5 \mathrm{sec}$ (slit-scan mode); the slit was transported at a uniform velocity through a slot by a Bodine variable-speed motor. By means of earphones, subjects were exposed to white noise throughout the experiment; its intensity was adjusted by each subject to mask any possible auditory cues from the Optacon display. The visual monitor (Model V1A) of the Optacon display was used to aid the experimenter in presenting the stimuli and to provide subjects with feedback between trials.

\section{Stimuli}

The stimuli were the uppercase Helvetica Light Condensed letters (Letraset 3115 ), excluding $M$ and W. Negatives were made of the 24 letters, their average dimensions (the letter I not included) being $5.8 \mathrm{~cm}$ high, $3.4 \mathrm{~cm}$ wide, and $.6 \mathrm{~cm}$ in stroke width. The zoom lens of the Optacon camera was set to either of two positions, resulting in two sizes of letters on the Optacon 
display. The display was comprised of 144 bimorph stimulators, arranged in 24 rows on $1.17-\mathrm{mm}$ centers and in six columns on 2.4-mm centers. The large letters were 18 rows $(2.0 \mathrm{~cm})$ high, five or six columns $(1.0$ or $1.2 \mathrm{~cm})$ wide, and two to three stimulators in stroke width. The small letters were 12 rows $(1.3 \mathrm{~cm})$ high, three or four columns $(.7$ or $1.0 \mathrm{~cm})$ wide, and one to two stimulators in stroke width. The slit width during slit-scan presentation was equal to the stroke width. The left index finger was placed over the vibrotactile display such that both large and small letters were centered just slightly distal to the joint separating the distal and medial segments of the finger. The intensity of the vibrotactile display was set so that when the bimorphs were active, the peak bimorph drive supply voltage was $30 \mathrm{~V}$. This was an intensity close to that used by Craig (Note 2).

\section{Subjects}

Four sighted females ranging in age from 18 to 23 years participated as paid subjects. None of the four had prior experience as psychophysical observers. The only criterion for each person's participation in the study was that she judge herself to be conscientious. All were naive as to the purpose of the study.

\section{Procedure}

Each subject participated for 30 1-h sessions, during each of which the subject received all 24 letters within each of the four conditions. The conditions were changed after each block of 13 letters. The orders of conditions and letters within conditions were random.

On a given trial, the subject was given a warning signal prior to the presentation of a letter. During the presentation, the subject's eyes were closed. Following this, the same letter was presented a second time in the same mode (static or slit-scan) while the subject viewed the visual monitor and felt the vibrotactile display. This procedure was adopted to allow the subject the best opportunity of learning to use the stimulus information being presented in the vibrotactile display.

\section{RESULTS}

The recognition scores (percent correct), averaged

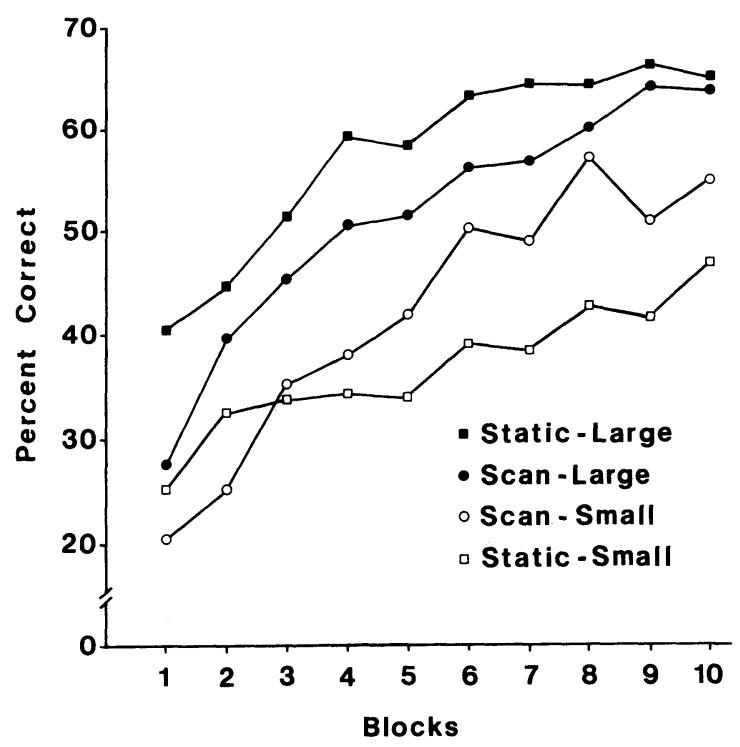

Figure 1. The percent correct recognition scores, averaged over subjects, as a function of experimental condition and each block of three sessions.
Table 1

Recognition Scores (Percent Correct) Averaged Over the Second Half of the Experiment

\begin{tabular}{cccccc}
\hline & \multicolumn{2}{c}{ Large Letters } & & \multicolumn{2}{c}{ Small Letters } \\
\cline { 2 - 3 } \cline { 5 - 6 } Subject & Static & Slit-Scan & & Static & Slit-Scan \\
\hline A.C. & 53.9 & 61.4 & & 26.1 & 47.2 \\
K.L. & 61.9 & 60.8 & & 31.9 & 53.9 \\
L.R. & 72.2 & 47.2 & & 49.4 & 41.7 \\
M.O. & 71.1 & 71.7 & & 59.7 & 67.2 \\
Mean & 64.8 & 60.3 & & 41.8 & 52.5 \\
\hline
\end{tabular}

over the four subjects, are given in Figure 1. The recognition scores within each block of three sessions were averaged for each subject, to simplify their presentation. The basic result is clear: After subjects became practiced, mode of stimulus presentation interacted strongly with character size. Whereas performance with the static mode dropped considerably with the decrease in character size, as one would expect if spatial resolution were limiting for smaller characters, much less of a decline was observed with slit-scan presentation. Furthermore, the averaged data indicate that whereas the static mode enjoyed a slight advantage with the larger letters, as Craig (Note 2) found, the slit-scan mode was considerably better with small letters.

The picture is not quite so simple when the data of the individual subjects are examined. Table 1 gives the average recognition score for each subject in each condition for the second half of the experiment. The interaction between display mode and character size is readily apparent for all four subjects. However, the superiority of the static mode for large letters was true only for one subject, and the two modes were roughly equal for two of the others. The superiority of the slitscan mode for small letters is more apparent; two subjects performed much better with it, one slightly better, and one slightly worse.

The present experiment would have been a more decisive test if even smaller letters could have been used, but the limited display resolution and the difficulty in maintaining proper registration between the letters and the photodiode array of the camera precluded this, for smaller letters would have suffered a significant loss in fidelity. Computer control of the Optacon display, the route taken by Craig (1979, Note 2), would have avoided the registration problem, but it was not an available option in this work. An assessment of whether the optically produced stimuli in this experiment were of adequate fidelity was carried out by having the four subjects attempt to identify the letters by viewing the visual monitor alone, this being done after Hour 20 of the tactile experiment. For the large and small letters presented statically, the average recognition scores were $100 \%$ and $97 \%$, indicating that stimulus quality was satisfactory. These same scores were $91 \%$ and $92 \%$ for the large and small letters displayed by slit-scan; these lower scores reflect the slight difficulty subjects have in integrating the information in the rapidly changing 
display, difficulty that must be a factor in the tactile recognition task as well.

\section{DISCUSSION}

Loomis (1974) proposed that the superiority of sequential presentation found with the TVSS was understandable in terms of the very poor cutaneous spatial resolution of the back. The basic argument may be stated as follows. Because of the poor spatial resolution of the back, even large letters presented statically are low-pass filtered of much of the critical information necessary for recognition. However, if the patterns are slit-scanned, or, better yet, scanned by a point aperture prior to display (and the cutaneous low-pass filtering), more information is necessarily available for the cortical stages involved in the processing of form. This can be easily appreciated by considering scanning with an infinitesimally narrow point aperture. The resulting neural-spread function corresponding to the product of the aperture and the pattern varies in amplitude and position in accordance with the light distribution of the pattern and the location of the aperture. In principle, all the pattern information is transmitted to cortical levels and is thus available for reconstruction of the pattern. In actuality, however, this information is not fully utilized, either because of subsequent distortions in cutaneous processing (Kirman, 1978) or because of the limitations of memory and other cognitive processes. It is probably correct to say that only when spatial resolution is severely limiting and the patterns are relatively simple does the greater information transmission of sequential presentation outweigh its limitations and lead to better recognition performance than is possible with simultaneous display of the pattern. If correct, this might explain why Sherrick (in press) did not find any superiority of sequential presentation in a task in which lines presented to the thigh by means of a vibrotactile display had to be discriminated in terms of orientation. The high performance levels (close to $80 \%$ correct) would indicate that spatial resolution was not a prominent limiting factor.

What Craig (Note 2) has shown is that this earlier result obtained with the TVSS is not found with the Optacon as it is normally used; indeed, he found the opposite result. This result is significant for at least two reasons. (1) The limitedresolution hypothesis (Loomis, 1974) does not apply to the recognition of large roman letters displayed on the finger using the Optacon. (2) In more practical terms, this finding in conjunction with Craig's (1979) earlier work demonstrates that the static display mode leads to the best recognition performance possible with the Optacon.

The present finding, although of little practical significance, affirms the basic correctness of Loomis' (1974) argument. Even though the limited-resolution hypothesis (Loomis, 1974) does not apply to the recognition of large letters with the Optacon, it does predict that if letters are reduced to a size such that their spatial bandwidths significantly exceed the bandwidth of the finger, more information will be available through sequential presentation. The present work provides evidence consonant with this prediction.

The finding here of a strong interaction between display mode and character size is pertinent to an earlier study. Apkarian-Stielau and Loomis (1975) attempted to simulate tactile letter recognition with the TVSS using blurred vision. Although they established several similarities between the two modalities, they were unable to show the superiority of slitscan presentation with blurred vision that they had expected. However, both static and slit-scan recognition scores for blurred vision were close to $65 \%$, whereas the tactile scores were significantly lower ( $30 \%$ and $45 \%$, respectively). Had they used smaller letters in the vision task (or, as they suggested, increased the degree of blur), it is quite likely that the superiority of slit-scan display would have been the result, by analogy to the present finding.

\section{REFERENCE NOTES}

1. Saida, S., Shimizu, Y., \& Wake, T. Construction of small TVSS and optimal mode of stimulus presentation. Paper presented at the Fourth Symposium on Sensory Substitution, Tokyo, Japan, November 1978.

2. Craig, J. C. Tactile letter recognition: Pattern duration and modes of pattern generation. Manuscript in preparation, 1980.

3. Kirman, J. H. Tactile pattern perception and tactile displays. Paper presented at the Conference on Interrelations of the Communicative Senses, Asilomar, California, September 1978.

\section{REFERENCES}

Apkarian-Stiflau, P., \& Loomis, J. M. A comparison of tactile and blurred visual form perception. Perception \& Psychophysics, $1975,18,362-368$

Beauchamp, K. L., Matheson, D. W., \& Scadden, L. A. Effects of stimulus-change method on tactile-image recognition. Perceptual and Motor Skills, 1971, 33, 1067-1070.

Craig, J. C. Modes of vibrotactile pattern generation. Journal of Experimental Psychology: Human Perception and Performance, 1979, 6, 151-166.

Loomis, J. M. Tactile letter recognition under different modes of stimulus presentation. Perception \& Psychophysics, 1974, 16, 401-408.

Sherrick, C. E. Cutaneous communication. In W. D. Neff (Ed.), Contributions to sensory physiology. New York: Academic Press, in press.

(Received for publication October 1, 1980.) 